\title{
Home enteral nutrition for postoperative elderly patients with esophageal cancer
}

\author{
Xueyu Chen, Guangyin Zhao, Lianggang Zhu \\ Department of Thoracic Surgery, Ruijin Hospital, Shanghai Jiaotong University School of Medicine, Shanghai, China \\ Contributions: (I) Conception and design: X Chen; (II) Administrative support: None; (III) Provision of study materials or patients: None; (IV) \\ Collection and assembly of data: X Chen, G Zhao; (V) Data analysis and interpretation: X Chen, G Zhao; (VI) Manuscript writing: All authors; (VII) \\ Final approval of manuscript: All authors. \\ Correspondence to: Lianggang Zhu, MD. Associate Chief Physician, Department of Thoracic Surgery, Ruijin Hospital, Shanghai Jiaotong University \\ School of Medicine, Shanghai 201801, China. Email: rjhnxw@163.com.
}

\begin{abstract}
Backgrounda The clinical value of enteral nutrition (EN) after radical resection of esophageal cancer (EC) has been well recognized during hospital stay; however, whether using EN agents should be continued at home after the patient is discharged remains unclear, especially for the elderly postoperative patients. Here we investigated the effects of continued EN on nutrition and immune status in elderly patients who had undergone radical EC surgery.

Methods: Sixty eligible elderly patients undergoing surgical treatment for EC in our center during the period from October 2016 to October 2018 were randomly divided into EN group and control groups, with 30 patients in each group. Among them, the EN group continued to take an orally administered EN agent (Ensure $^{\circledR}$ ) daily in addition to daily routine diets after discharge; however, patients in the control group only received regular diets after discharge. The nutritional status and immune indicators were evaluated at discharge and 4 and 8 weeks after discharge (weeks 4 and 8) and compared between EN and control groups.

Results: Body mass index (BMI), Patient-Generated Subjective Global Assessment (PG-SGA) score, hemoglobin, serum albumin, serum prealbumin, CD4 and CD8 T cell counts, CD4/CD8 ratio, IgA, IgG, and IgM showed no significant difference between EN group and control group at discharge (all $\mathrm{P}>0.05$ ). In week 4, the serum prealbumin level was significantly higher in the EM group than in the control group $(\mathrm{P}<0.05)$. In week eight, the EM group had significantly higher BMI, PG-SGA score, serum albumin, serum prealbumin, CD4 and CD8 T cell counts, CD4/CD8 ratio, IgA, IgG, and IgM than the control group (all $\mathrm{P}<0.05)$.
\end{abstract}

Conclusions: Home EN helps improve immune function in elderly patients who have undergone radical surgery for EC and is worthy of clinical promotion. To optimize its efficacy, a home EN should last no less than eight weeks after discharge.

Keywords: Elderly patients; esophageal cancer (EC); enteral nutrition (EN); nutritional status; cellular immunity; humoral immunity

Submitted Oct 15, 2020. Accepted for publication Dec 26, 2020.

doi: 10.21037/apm-20-2197

View this article at: http://dx.doi.org/10.21037/apm-20-2197

\section{Introduction}

Esophageal cancer (EC) is still one of the most common malignancies worldwide. In China, the crude incidence rate of EC was 278.07/100,000, and the crude mortality rate was $167.89 / 100,000$ in 2014 , both ranking first in the world (1). EC patients often have a problem swallowing (known as dysphagia), which, coupled with the tumor's consumption, results in nutritional problems. Particularly, elderly EC patients have a weak ability to absorb nutrients, and dysphagia and surgical trauma further cause a sharp 
decline in postoperative nutrition, immune function, and life quality. Focusing on the elderly patients with malignant esophageal tumors, we investigated the effects of home EN on postoperative nutritional status and immune function in these patients and explored its clinical values. We present the following article in accordance with the CONSORT reporting checklist (available at http://dx.doi.org/10.21037/apm-20-2197).

\section{Methods}

\section{Subjects}

The Ruijin Hospital Shanghai Jiaotong University School of Medicine gave ethical approval. All procedures performed in this study involving human participants were in accordance with the Declaration of Helsinki (as revised in 2013). Because of the retrospective nature of the research, the requirement for informed consent was waived.

Patients aged $\geq 60$ years who underwent radical surgery for esophageal malignancies in our department from October 2016 to October 2018 were consecutively enrolled. All the subjects met the diagnostic criteria described in the Clinical Practice Guidelines for the Diagnosis and Treatment of Esophageal Cancer (2).

The inclusion criteria were as follows: (I) an esophageal neoplasm is observed under electronic gastroscope and is pathologically confirmed as a malignant esophageal tumor; (II) preoperative cardiopulmonary function tests show no obvious abnormality, and the patient can tolerate the surgery; (III) preoperative contrast-enhanced phased computed tomography (CT), Doppler color ultrasonography, and/or PET-CT reveal that the clinical stage is below T3N0M0; and (IV) there is no history of diabetes, gastrointestinal disease, or gastrointestinal surgery; and $(\mathrm{V})$ aged $\geq 60$ years.

The exclusion criteria included: (I) patients requiring radiochemotherapy with their postoperative pathological stage; (II) patients with digestion problems who cannot tolerate oral nutritional supplements, not improved after medication use; (III) patients who experience delayed discharge due to postoperative complications including anastomotic leak; (IV) patients who have mild anastomotic stenosis after surgery, which causes difficulty swallowing when eating regular diets; and $(\mathrm{V})$ patients who have diabetes, metabolic diseases, and/or other gastrointestinal diseases may cause nutritional disorders.

According to the above inclusion and exclusion criteria, 64 subjects (including 56 men and eight women) were included in the first analysis. These subjects aged 61-75 years, and squamous cell carcinoma was pathologically confirmed in all patients. After the operation, two patients were noted to have delayed anastomotic leaks, one patient developed severe diarrhea after using an enteral nutrition (EN) agent, and one patient could not eat regular diets due to postoperative anastomotic stenosis. These four subjects withdrew from our research. According to the random assignment principle after the operation, the remaining 60 patients were divided into the EN group ( $n=30)$ and the control group $(n=30)$.

\section{Intervention}

All the included patients underwent radical surgery for EC by thoracic surgeons from the same treatment team following the Guidelines for Standardized Diagnosis and Treatment of Esophageal Cancer (2). Depending on tumor location, patients with upper EC were treated with thoracic laparoscopy combined with EC resection and neck anastomosis (known as McKeown procedure). Those with middle or lower EC were treated with a laparoscopically assisted two-field abdominal-thoracic operation (Ivor-Lewis procedure). During the operation, nasointestinal tubes were indwelled in all patients; on the first postoperative day, $250 \mathrm{~mL}$ of warm water was given through the nasointestinal tube to enable gut adaptation. EN was offered via the nasointestinal tube after the second postoperative day, and the caloric intake was gradually increased to 2,000 calories/day. On the 7th to 10th postoperative days, a water-soluble contrast swallow was routinely performed for anastomosis assessment. If there was no anastomotic leak, the patients began to drink a small amount of water, then transitioned to a semi-liquid diet. The regular diet was offered to both groups after discharge; in the EN group, patients continued to receive EC (EnSure ${ }^{\circledR}$, Abbott Laboratories, the Netherlands) daily with or between meals as we suggested, six standard scoops of EnSure were dissolved in $200-250 \mathrm{~mL}$ warm water before they were slowly orally swallowed. The frequency was no less than 6 times/day. Whether or not the patient in En group will continue to use enteral nutrition preparations after 8 weeks will be determined by each patient according to their own feeding status.

However, patients in the control group only received regular diets after discharge. The demographic data of all subjects were collected. Body fat index (BMI) and the Patient-generated Subjective Global Assessment (PG-SGA) score were measured before discharge and 4 and 8 weeks after discharge (weeks 4 and 8). PG-SGA scores, assessed by both patients and medical staff, can reflect nutritional status (3). 
Table 1 General data in two groups

\begin{tabular}{lcc}
\hline & EN group $(\mathrm{n}=30)$ & Control group $(\mathrm{n}=30)$ \\
\hline Age (years) & $70.37 \pm 7.58$ & $69.53 \pm 9.49$ \\
Sex (female/male) & $5: 25$ & $3: 27$ \\
Ivor-Lewis procedure & 24 & 25 \\
McKeown procedure & 6 & 5 \\
Postoperative stage ( $)$ & 5 & 4 \\
Stage IIB & 12 & 15 \\
Stage IIA & 13 & 11 \\
Stage IIB & $11.5 \pm 2.58$ & $12.7 \pm 2.87$ \\
Postoperative hospital stay (days) & & 15 \\
\hline
\end{tabular}

Also, hemoglobin, serum albumin, serum prealbumin, CD4, and CD8 $\mathrm{T}$ cell counts, and immunoglobulin (IgA, IgM, and $\operatorname{IgG}$ ) levels were determined.

\section{Collection of data and specimens}

The PG-SGA form was distributed to the subjects one day before the assessment and collected the following morning. Patients with a PG-SGA score of $0-3$ were regarded as nonmalnourished and those with a score of $\geq 4$ as malnourished. The same group of medical staff obtained BMI data. At 6 a.m., a nurse collected $10 \mathrm{~mL}$ of fasting peripheral venous blood from the patient to determine hemoglobin, serum albumin, serum prealbumin, CD4, and CD8 T cell counts immunoglobulin (IgA, IgM, and IgG) levels.

\section{Statistical analysis}

Statistical analysis was performed in the PASW Statistics 18.0 software. All the samples were evaluated for normality, and all the parameters are presented with range. The normally distributed parameters are described as mean \pm standard deviation. The measurement data were compared using the $t$-test for two independent samples; for count data, the rates were for the $\chi^{2}$ test on a fourfold table. A P value of less than 0.05 was statistically significant.

\section{Results}

\section{Basic information}

There were 25 males and five females in the EN group and 27 males and three females in the control group. These two groups showed no statistical significance in sex composition, average age, surgical procedures, postoperative pathological type, postoperative pathological stage, and postoperative hospital stay (Table 1).

\section{Nutritional status and immune function at discharge}

Nutritional status and immune function showed no significant difference between EN and control groups at discharge (Table 2).

\section{Nutritional status and immune function in weeks 4 and 8}

In week 4, BMI, hemoglobin, serum albumin, serum prealbumin, and various cellular and humoral immune indicators were improved in the EN group (compared with those before discharge). PG-SGA revealed three patients in the EN group judged to be malnourished at discharge became non-malnourished in week 4 . In the control group, the nutrition and immune indicators in week 4 were slightly lower than those at discharge, and only IgA and serum prealbumin were slightly improved compared with those before discharge. Also, one patient in the control group was newly identified as malnourished on the PG-SGA scale. Comparisons between these two groups in week 4 showed that the EN group had superior serum prealbumin levels to the control group, and the difference was statistically significant.

In week eight, all the nutrition and immune indicators were further improved in the EN group. As shown on the PG-SGA scale, there were only three malnourished patients in the EN group, and the proportion of patients with 
Table 2 Comparisons of parameters reflecting nutritional status and immune function between two groups at discharge

\begin{tabular}{lcc}
\hline & EN group $(\mathrm{n}=30)$ & Control group $(\mathrm{n}=30)$ \\
\hline BMI $\left(\mathrm{kg} / \mathrm{m}^{2}\right)$ & $17.4 \pm 2.1$ & $17.7 \pm 2.0$ \\
PG-SGA score (points) & & 20 \\
Malnourished & 22 & 10 \\
Non-malnourished & 8 & $112.1 \pm 8.5$ \\
Hemoglobin $(\mathrm{g} / \mathrm{dL})$ & $108.6 \pm 10.4$ & $30.3 \pm 3.9$ \\
Serum albumin $(\mathrm{g} / \mathrm{L})$ & $30.7 \pm 4.7$ & $178.6 \pm 33.5$ \\
Serum prealbumin $(\mathrm{g} / \mathrm{L})$ & $182.0 \pm 28.4$ & $34.6 \pm 4.7$ \\
CD4 (g/L) & $33.4 \pm 5.4$ & $31.2 \pm 4.8$ \\
CD8 $(\mathrm{g} / \mathrm{L})$ & $30.5 \pm 3.3$ & $1.15 \pm 0.32$ \\
CD4/CD8 (g/L) & $1.12 \pm 0.40$ & $10.11 \pm 4.66$ \\
$\operatorname{lgG}(\mathrm{g} / \mathrm{L})$ & $9.76 \pm 5.50$ & $1.83 \pm 0.58$ \\
$\operatorname{lgA}(\mathrm{g} / \mathrm{L})$ & $1.85 \pm 0.45$ & $1.67 \pm 0.49$ \\
IgM (g/L) & $1.77 \pm 0.47$ &
\end{tabular}

BMI, body mass index; PG-SGA, Patient-Generated Subjective Global Assessment; EN, enteral nutrition.

malnutrition was significantly lower than that in the control group. Furthermore, the EN group had superior BMI, serum prealbumin, serum albumin, and all immune markers to the control group, and the differences were statistically significant (Table 3).

After 8 weeks of observation, all the patients in the EN group well tolerated the use of enteral nutrition preparations. No patients in EN group had related adverse reactions and were unable to continue using enteral nutrition because of intolerance. We believe that the use of home EN in has shown satisfactory safety and feasibility.

\section{Discussion}

EC is one of the most common malignant tumors of the digestive tract in China. Globally, there were about 500,000 new EC cases diagnosed and 400,000 deaths from EC in 2012 (4). About $50 \%$ of the world's EC incidence and mortality rates are noted in China $(1,5)$. With an attempt to improve the surgical cure of EC in elderly patients, various minimally invasive and endoscopic surgical procedures for malignant esophageal tumors have been described in recent years, focusing on reducing complications and achieving rapid recovery (6-8). While some foreign studies have reported the nutritional support for recovery from surgery for EC in elderly patients (9), few Chinese articles have investigated this topic. In elderly patients with EC, the digestive system's secretion and absorption functions have already declined with age, and the traumatic stress of surgery and the postoperative changes in eating patterns and habits further impair the absorption of nutrients after surgery (10). All these factors hinder the restoration of postoperative nutritional status. Chen et al. (11) conducted preoperative nutritional risk screening in $180 \mathrm{EC}$ patients and found that the incidence of malnutrition in patients older than 60 years was higher than that in those younger than 60 years. Wu et al. (12) also carried out nutritional risk screening in over 4,000 inpatients in the general surgery department at Shanghai Zhongshan Hospital. They found the elderly (60 years old or above) patients with gastrointestinal malignancies had significantly higher malnutrition risk than those who were younger than 60 years or those without gastrointestinal tumors. Another Chinese study enrolling 2,500 elderly inpatients showed about $30 \%$ of elderly hospitalized patients had varying degrees of malnutrition (13). These findings have justified that elderly patients undergoing surgery need nutritional support during the perioperative period and after discharge.

We chose EnSure as an enteral nutrition preparation after discharge for patients with esophageal cancer mainly because of its following advantages: (I) high cost performance, comprehensive and balanced nutrition, easy 
Table 3 Nutritional status and immune function in two groups 4 and 8 weeks after discharge

\begin{tabular}{|c|c|c|c|c|}
\hline & \multicolumn{2}{|c|}{ Week 4} & \multicolumn{2}{|c|}{ Week eight } \\
\hline $\mathrm{BMI}\left(\mathrm{kg} / \mathrm{m}^{2}\right)$ & $17.9 \pm 2.2$ & $16.3 \pm 1.8$ & $19.1 \pm 4.8^{8}$ & $16.1 \pm 4.3$ \\
\hline \multicolumn{5}{|l|}{ PG-SGA score (points) } \\
\hline Malnourished & 19 & 21 & $3^{\& \#}$ & 15 \\
\hline Hemoglobin (g/dL) & $115.1 \pm 9.4$ & $108.9 \pm 11.3$ & $135 \pm 7.1^{\#}$ & $127 \pm 8.4$ \\
\hline Serum albumin (g/L) & $32.3 \pm 4.2$ & $30.2 \pm 5.0$ & $40.1 \pm 5.9^{\& \#}$ & $31 \pm 3.8$ \\
\hline Serum prealbumin $(g / L)$ & $248.5 \pm 31.3^{*}$ & $198.6 \pm 37.1$ & $325 \pm 45.6^{\text {\&\# }}$ & $225 \pm 51.6$ \\
\hline CD4 (g/L) & $37.6 \pm 6.2$ & $33.1 \pm 7.1$ & $40.6 \pm 8.6^{8 \#}$ & $31.3 \pm 10.5$ \\
\hline $\operatorname{lgG}(g / L)$ & $11.36 \pm 4.20$ & $10.8 \pm 3.46$ & $15.2 \pm 5.68^{\& \#}$ & $10.9 \pm 4.61$ \\
\hline $\lg A(g / L)$ & $1.98 \pm 0.61$ & $1.92 \pm 0.41$ & $3.0 \pm 0.98^{\& \#}$ & $1.84 \pm 0.67$ \\
\hline $\lg M(g / L)$ & $1.97 \pm 0.38$ & $1.64 \pm 0.43$ & $2.48 \pm 0.79^{\&}$ & $1.69 \pm 0.59$ \\
\hline
\end{tabular}

${ }^{*} \mathrm{P}<0.05$, compared with the control group in week $4 ;{ }^{\circledR} \mathrm{P}<0.05$, compared with the control group in week eight; $\mathrm{P}<0.05$, comparison between week eight and at discharge in EN group. BMI, body mass index; PG-SGA, Patient-Generated Subjective Global Assessment; EN, enteral nutrition.

to absorb by body; (II) good intestinal tolerance; (III) good taste. In our current study, patients in both groups have different hypoproteinemia degrees, anemia, low body weight, and other manifestations associated with poor nutritional status at once after discharge. In week 4, BMI, PG-SGA score, hemoglobin, and serum prealbumin level were improved in the EN group (compared with the measurements at discharge). However, in the control group, the improvements in these nutritional evaluation indicators were not as obvious as in the EN group. Even worse, the PG-SGA score, BMI, serum albumin, and hemoglobin further decreased in the control group due to the persisting negative nitrogen balance caused by surgical trauma-induced hypermetabolism. Although only the serum prealbumin showed a significant difference between these two groups in week 4, the effects of postoperative continuous EN on the postoperative nutritional status became visible. In week eight, the above data were further compared between the EN group and the control group. BMI, PG-SGA score, serum prealbumin, and serum albumin levels were significantly higher in the EN group than in the control group, suggesting 4-8 weeks of EN in elderly patients with EC improved the nutritional status. Further comparisons of the data at discharge and in week 8 in the EN group showed that most of the nutritional indicators were superior in week 8 than at discharge, and the differences were statistically significant. Thus, we recommend offering at least 8 weeks of continuous EN support for elderly patients undergoing surgery for EC after discharge.

Also, we have observed that EC patients typically have weakened the immune system following malnutrition. In the control group, the CD4 count and CD4/CD8 ratio were slightly lower in week four than at discharge. Surgical stress and trauma, perioperative complications, and postoperative hyponutritional status are important reasons for the decline of postoperative immune function in EC patients, especially in elderly ones (14). The decreased immune function directly affects immune cells' ability to recognize and kill tumor cells, leading to tumor recurrence and progression (15). In healthy individuals, the $\mathrm{T}$ cell subsets support the normal immune function, and $\mathrm{CD} 4+$ and $\mathrm{CD} 8+\mathrm{T}$ cells are the whole $\mathrm{T}$ cells. CD4+ $\mathrm{T}$ cells participate in immune memory response and can directly kill tumor cells; yet, CD8+ T cells are suppressor $\mathrm{T}$ cells that coordinate with $\mathrm{CD} 4+\mathrm{T}$ cells to participate in cellular immunity $(16,17)$. Lymphocyte dysfunction has been identified in research on peripheral blood CD4, CD8, and CD4/CD8 in tumor patients. It has been found that the T-cell subset proportions can reflect 
the immune status in tumor patients (18). Impaired cellular immunity is manifested as a decrease in circulating CD4 $\mathrm{T}$ cells, a relative increase in CD8 $\mathrm{T}$ cells, and a decrease in the CD4/CD8 ratio $(19,20)$. In our current study, CD4 count, CD 8 count, and CD4/CD 8 ratio increased in the EN group in both weeks 4 and week 8 , and CD 4 count and CD4/CD8 ratio were significantly higher in the EN group than in the control group in week 8 (both $\mathrm{P}<0.01$ ).

Similarly, immunoglobulins (Igs) are an important part of the immune defense against pathogens and play key roles in immunosurveillance and anti-tumor and anti-infection host defense. In our current study, the humoral immune indicators, including $\operatorname{IgG}, \operatorname{IgA}$, and $\operatorname{IgM}$, were superior in the EN group to the control group in week 8. Also, we found that some immune indicators were significantly improved after 8 weeks of continuous home EN support. Therefore, in addition to nutritional benefits, 8 weeks of extra EN in elderly EC patients after discharge can also improve the cellular immunity and humoral immunity, improve the postoperative quality of life, and increase disease response rates.

While the value of perioperative $\mathrm{EN}$ in $\mathrm{EC}$ patients has been widely recognized $(21,22)$, its application should not be limited to the period of hospitalization in surgical EC patients, especially in elderly ones. In our current study, the continued use of EN agents after discharge in elderly EC patients undergoing surgery significantly improved nutritional status and played an important role in enhancing cellular and humoral immunity; further, its small sample size limited our study. Also, only early EC patients who did not need postoperative adjuvant treatment were enrolled. We strictly limited the criteria of patient inclusion and exclusion, in order to minimize the impact of unrelated factors on the results of the study, so as to ensure the reliability of the results of the study. Therefore, after strict screening, the sample size of patients who eventually entered our study was relatively small, but our preliminary study showed that meaningful clinical experimental results have been reached. Nevertheless, our findings may also be applicable for patients with advanced EC who need better nutrition and immune status and require postoperative radiotherapy and chemotherapy. It is hoped this nutritional support concept will be extended to all postoperative EC and non-surgical EC patients in the future.

\section{Acknowledgments}

We acknowledge the Ruijin Hospital, Shanghai Jiaotong
University School of Medicine.

Funding: None.

\section{Footnote}

Reporting Checklist: The authors have completed the CONSORT reporting checklist. Available at http://dx.doi. org/10.21037/apm-20-2197

Data Sharing Statement: Available at http://dx.doi. org/10.21037/apm-20-2197

Conflicts of Interest: All authors have completed the ICMJE uniform disclosure form (available at http://dx.doi. org/10.21037/apm-20-2197). The authors have no conflicts of interest to declare.

Ethical Statement: The authors are accountable for all aspects of the work in ensuring that questions related to the accuracy or integrity of any part of the work are appropriately investigated and resolved. The Ruijin Hospital Shanghai Jiaotong University School of Medicine gave ethical approval. All procedures performed in this study involving human participants were in accordance with the Declaration of Helsinki (as revised in 2013). Because of the retrospective nature of the research, the requirement for informed consent was waived.

Open Access Statement: This is an Open Access article distributed in accordance with the Creative Commons Attribution-NonCommercial-NoDerivs 4.0 International License (CC BY-NC-ND 4.0), which permits the noncommercial replication and distribution of the article with the strict proviso that no changes or edits are made and the original work is properly cited (including links to both the formal publication through the relevant DOI and the license). See: https://creativecommons.org/licenses/by-nc-nd/4.0/.

\section{References}

1. Sun KX, Zheng RS, Zhang SW, et al. Report of Cancer Incidence and Mortality in Different Areas of China, 2015. China Cancer 2018;28:1-14.

2. Chinese Society of Esophageal Cancer Chinese AntiCancer Association. Clinical Practice Guidelines for the Diagnosisi and treatment of esophageal cancer. Beijing: Peking Union Medical College Press; 2013 Apr. 14p.

3. Bauer J, Capra S, Ferguson M. Use of the scored Patient- 
Generated Subjective Global Assessment (PG-SGA) as a nutrition assessment tool in patients with cancer. Eur J Clin Nutr 2002;56:779-85.

4. Jemal A, Bray F, Center MM, et al. Global cancer statistics, 2012. CA Cancer J Clin 2011;61:69-90.

5. Zhang M, Zhang C, Wu QC. Health-related quality of life and survival among 10-year survivors of esophageal cancer surgery: gastric tube reconstruction versus whole stomach reconstruction. J Thorac Dis 2019;11:3284-91.

6. Qi W, Zixiang W, Tianwei Z, et al. Long-term outcomes of 530 esophageal squamous cell carcinoma patients with minimally invasive Ivor Lewis esophagectomy. J Surg Oncol 2018;117:957-69.

7. Liu W, Guo X, Zhao H, et al. Mediastinoscopy-assisted transhiatal esophagectomy versus thoraco-laparoscopic esophagectomy for esophageal cancer: a single-center initial experience. J Thorac Dis 2020;12:4908-14.

8. Park SY, Kim DJ, Yu WS, et al. Robot-assisted thoracoscopic esophagectomy with extensive mediastinal lymphadenectomy: experience with 114 consecutive patients with intrathoracic esophageal cancer. Dis Esophagus 2016;29:326-32.

9. Bowrey David J, Baker Melanie, Halliday Vanessa, et al. A randomised controlled trial of six weeks of home enteral nutrition versus standard care after oesophagectomy or total gastrectomy for cancer:report on a pilot and feasibility study. Trials 2015;16:531-43.

10. Grace EM, Shaw C, Lalji A, et al. Nutritional status, the development and persistence of malnutrition and dietary intake in oesophago-gastric cancer:a longitudinal cohort study. J Hum Nutr Diet 2018;31:785-92.

11. Chen XS, Chen FX, Huang XM. Analysis of preoperative nutritional risk in 180 patients with esophageal cancer. Chongqing Medicine 2014;43:1363-5.

12. Wu GH, Liu ZH, Zheng LW, et al. Prevalence of malnutrition in general surgical patients:evaluation of nutritional status and prognosis. Zhonghua Wai Ke Za Zhi 2005;43:693-6.

13. Tang DN, Wei JM, Zhu MW, et al. Prevalence of nutritional risk and malnutrition and nutrition support in elderly hospitalized patients. Chinese Journal of Geriatrics
2011;30:974-6.

14. Wu GH. Nutritional support in patients with tumors Causes and hazards of malnutrition in patients with tumors. Chinese Journal of Practical Surgery 2002;22:644-6.

15. Othman MO, Lee JH, Wang K. Clinical Practice Update on the Utility of Endoscopic Submucosal Dissection in T1b Esophageal Cancer: Expert Review. Clin Gastroenterol Hepatol 2019;17:2161-6.

16. Wang XY, Kazim Latif, Repasky Elizabeth A, et al. Immunization with tumor-derived ER chaperone grp170 elicits tumor-specific CD8+ T-cell responses and reduces pulmonary metastatic disease. Int $\mathrm{J}$ Cancer 2003;105:226-31.

17. Yang J, Huang ZY, Yuan Q, et al. Effect of General Anesthesia Combined with Epidural Anesthesia on T-lymphocyte Subsets in Elderly Patients with Esophageal Cancer in Peroperative Period. Journal of Clinical Research 2012;29:818-20.

18. Ostroumov D, Fekete-Drimusz N, Saborowski M, et al. CD4 and CD8T lymphocyte interplay in controlling tumor growth. Cell Mol Life Sci 2018;75:689-13.

19. Decker D, Schondorf M, Bidlingmaier F, et al. Surgical stress induces a shift in the type-1/type-2 T-helper cell balance, suggesting down-regulation of cell-mediated and up-regulation of antibody-mediated immunity commensurate to the trauma. Surgery 1996;119:316-25.

20. Tashiro T, Yamamori H, Takagi K, et al. Changes in immune function following surgery for esophageal carcinoma. Nutrition 1999;15:760-6.

21. Xu SB, Xu MQ, Sun XH, et al. The correlation of prognostic nutritional index with postoperative complications and prognosis in patients with esophageal cancer. Chinese Journal of Clinical Oncology 2018;45:789-94.

22. Ji Y, Chen JY, Zheng MF, et al. Clinical research on early postoperative enteral nutrition in patients with esophageal cancer. Parenteral \& Enteral Nutrition 2012;19:21-3.

(English Language Editor: J. Chapnick)
Cite this article as: Chen X, Zhao G, Zhu L. Home enteral nutrition for postoperative elderly patients with esophageal cancer. Ann Palliat Med 2021;10(1):278-284. doi: 10.21037/apm20-2197 